\title{
A CLINICAL AND PATHOLOGICAL STUDY OF THE HEART IN DIPHTHERIA
}

\author{
By WILLTAM E. HUME aNd S. J. CLEGG
}

With Plates 1 and 2

IN the summer of 1912, we commenced an investigation into the irregularities of the heart which occur in certain cases of diphtheria. From that time to the beginning of March, 1914, 573 cases of diphtheria were admitted to the City Hospital for Infectious Diseases, Newcastle-on-Tyne.

All these cases were under the daily supervision of one of us (S.J. C.), and when any irregularity of the heart and pulse was detected, polygraphic records were obtained. Other cases were similarly investigated if the general condition of the patient on admission suggested that the heart would be affected in a later stage of the disease, even though the rhythm of the heart was regular during the early stages. In this way we have obtained polygraphic records of seventy cases, of which thirty were found to be normal. Of the forty cases which presented irregularities, twenty-two showed the characteristics of a sinus arrhythmia and were associated with normal cardiac signs and symptoms.

The remaining eighteen cases presented many different types of heart irregularity and form the subject of this communication. Though the varieties of arrhythmia are great, the cases fall naturally into certain clinical groups. For instance, in Group I six cases are described which had many features in common: the patients were very ill from the onset of the disease, the hearts presented gross and varying irregularities of rhythm, and the pathological picture in four cases was identical.

In Group II nine cases are included which manifested a less serious clinical condition in the early stages than those in Group I ; although the patients were seriously ill at some period of their rather prolonged stay in the hospital, there was less variety in the types of arrhythmia, and in two fatal cases the pathological lesions of the heart were slight as compared with the gross changes found in the four instances in Group I.

In Group III three mild cases are described in which some cardiac irregularity occurred without any other sign of involvement of the heart.

\section{Group $I$.}

In this group are included six cases which presented many features in common. On admission the patients were found to be suffering from a severe attack of diphtheria. The membrane in each case was extensive and luxuriant, 
and the general manifestations of a severe toxaemia were present. There was great prostration, vomiting occurred frequently, and death took place in each instance before the fifteenth day. Each case in this group was at once recognized as presenting certain general features which called for an early and constant study of the heart.

The important clinical features of each case are shown in Table I, in which the daily rate of the heart and the types of arrhythmia are set forth in detail. Three of these cases have already been described in considerable detail in Heart, vol. v, No. 1, p. 25, and it is only intended to refer to them in so far as they present points of similarity or contrast with the other three cases.

The similarity which was found in the clinical and pathological pictures of the cases already published was present also in the three later cases, and a summary of the three earlier forms an efficient introduction to the more detailed description of the three later cases.

Cases $I, I I$, and III. All three children attended the same school and presumably contracted the disease from the same source. The duration of the illness, which terminated fatally in all three cases, was almost identical, as two of them died on the thirteenth day of illness and the third died on the fifteenth day of illness. The march of the disease from day to day gave rise to changes in the clinical picture which followed each other with such regularity that one description might have served for all three cases. The vomiting, pallor, gradual dilatation of the heart, the altered character of the heart-sounds, the types of arrhythmia, the subnormal temperature and the other manifestations of toxaemia were present in almost each particular in all three cases. This coincidence is particularly striking in the time of appearance of the various types of arrhythmia. Premature beats of the heart, which were proved to be of auricular origin in one case and probably also were so in the other two cases, appeared on the ninth day in two cases and in the third case on the seventh day. In two cases a nodal rhythm appeared on the tenth day and in the third case on the eighth day. A condition thought to be auricular flutter was noted in two of them, in one instance on the eleventh day and in the other on the twelfth day.

Though the general similarity between the first and the last three cases is great, the latter must be described in some detail in as far as the arrhythmia of the heart is concerned.

Case IV. Eva J., aged 7 years, was admitted to the hospital on the third day of illness, suffering from diphtheria. A swab had been taken and Klebs-Loeffer bacilli were found. 4,000 units of antitoxin were administered on admission, and next day a further dose of 6,000 units was given. The membrane extended over both tonsils, and there was bleeding from the throat and nose. The face was pale and anxious, and there was great prostration. On the fourth day purpuric spots appeared on the trunk and upper arms. Vomiting occurred early and the child died on the tenth day of illness. The apex beat of the heart was found to be in the nipple line on the fourth day, and the first sound of the heart heard at the apex was short and sharp. Polygraphic records, which were taken frequently between the third and the sixth days, revealed a normal rhythm. On the sixth day the curve shown in Fig. 1 was obtained.

The jugular tracing is occupied by single waves in each cardiac cycle, which appear quite regularly. The upstroke of the wave corresponds to the beginning of ventricular systole, and there is no evidence of an $a$ wave preceding it. 
It would seem probable that the auricles and ventricles are contracting simultaneously and that the focus of stimulation resides in the auriculoventricular node. The fourth peak in the jugular curve, however, is bifid, and the upstroke precedes the marking (4) by a greater interval than do the remainder of the upstrokes. In this cycle the auricular contraction slightly precedes the ventricular contraction and in this way the bifid top and the earlier appearance of the upstroke are accounted for. Tracings of this type were obtained throughout the sixth day. On the seventh day the rhythm of the heart changed, and the pulse was found to be completely irregular and Fig. 2 was obtained.

It is apparent that the radial pulse is completely irregular, that in the jugular curve there is a prominent wave at the beginning of systole $c$, and that

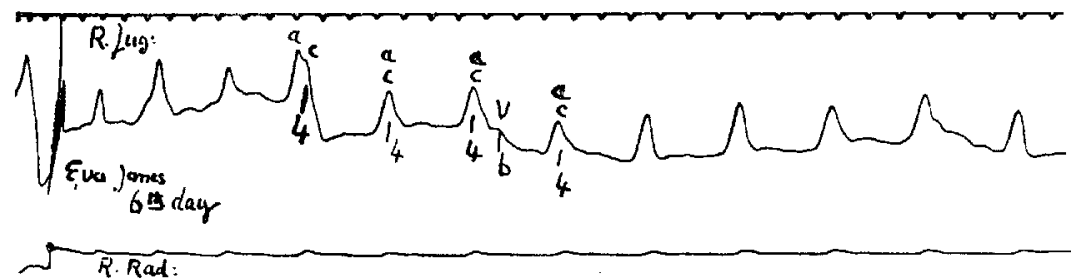

FIG. 1. Polygraphic curves from right jugular vein and right radial artery. In the jugular tracing there is a single large wave ac. In the fourth jugular cycle the wave is notched and the first peak is due to an auricular contraction. Rate $=100$.

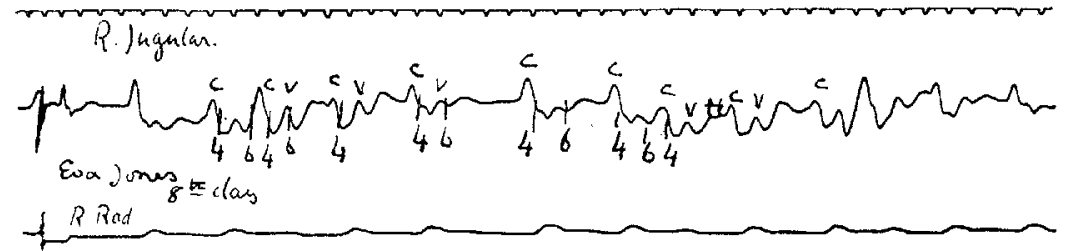

FIG. 2. Polygraphic curves from right jugular vein and right radial artery. Radial pulse is completely irregular. Ventricular form of venous pulse.

no wave $a$ indicative of auricular contraction precedes $c$. It would seem certain that this curve indicates auricular fibrillation. The next day (the 8th) identically the same curves were obtained, and so also up to midday on the ninth day of illness.

Case V. John M., aged 4 years, was admitted on the sixth day of illness in an advanced state of toxaemia. 4,000 units of antitoxin had been administered on the third day of illness before admission to the bospital. There was an extensive membrane over both tonsils and the soft palate. The child was pale, and prostration was great. The temperature on admission was $99.4^{\circ}$, on the seventh day $99^{\circ}$, and on the eighth day fell to $98^{\circ}$; between the ninth and eleventh days (the day of death) the temperature ranged between $97^{\circ}$ and $98^{\circ}$. The pulse rate was slow and perfectly regular throughout. It never dropped below 62 beats per minute, nor exceeded 70 beats per minute. Numerous tracings were obtained from the seventh to eleventh days, and Fig. 3 is representative of them.

The radial pulse was so small that it was impossible to obtain a record from it, and it was necessary to utilize the apical impulse as the index of the 
commencement of ventricular systole. It is apparent that the beginning of the ventricular upstroke corresponds with the first upstroke in the jugular curve $c$. This is followed by a $v$ wave. No wave indicative of auricular contraction precedes the wave marked $c$. The venous curve would appear to be open to three interpretations. In the first place the ventricular form of venous pulse and the regularity of the ventricles would point to the presence of auricular fibrillation and heart-block. A similar tracing with this interpretation has been published by Price and Ivy Mackenzie. In the second place the auricles may have been in a state of 'flutter' and the auricular contractions may have been too small to make any impression on the venous pulse. In the third place the auricles may be contracting at the same rate as the ventricles, but do so either simultaneously or later than the ventricles. The comparatively slow rate of the

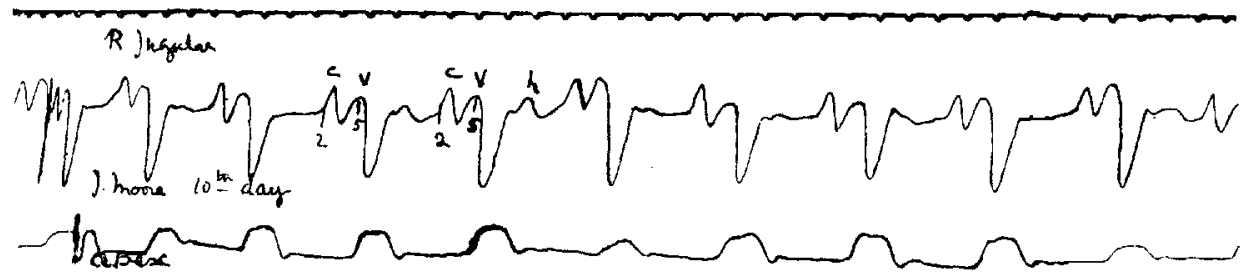

FIG. 3. Polygraphic curves from right jugular vein and apex beat. Ventricular systolic rise $(c)$ in jugular curve, followed by $(v)$ wave. Rate $=66$.

ventricles and the form of the jugular curve make it probable that the first is the correct interpretation. The rhythm in the following case was practically identical with the foregoing, and the same explanation probably holds in both cases.

Case VI. Violet T., aged 6 years, was admitted on the sixth day of illness and died on the eighth day. 2,000 units of antitoxin had been given on the fifth day and on admission 4,000 units were injected. The child was extremely ill, pale, prostrate and vomiting. The temperature was $97^{\circ}$ and the pulse rate was 72. On the seventh day the following polygraphic tracing was obtained (Fig. 4).

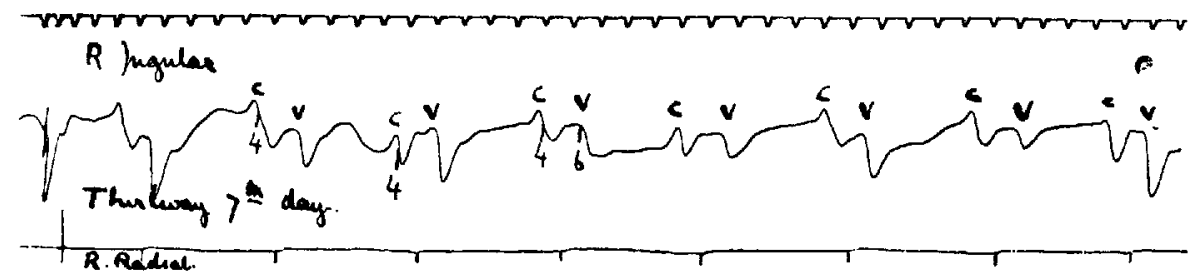

FrG. 4. Polygraphic curves from right jugular vein and right radial artery. Ventricular form of venous pulse. Rate $=60$.

The similarity of this curve to Tracing 3 is very great and the pulse rate is slightly slower-60 beats per minute. Of the alternative explanations the most probable would appear to be that there is fibrillation of the auricles together with complete auriculo-ventricular dissociation. The ventricular rate is faster in these instances of heart-block than it is in chronic heart-block in adults. Even in adults the idioventricular rate may reach 60 beats per minute, 
and there may be in children an idioventriculnr rate which naturally exceeds that of the adult.

Attention has already been called to the similarity which all the cases forming this group bear to each other. The infection was virulent, and in no case was antitoxin administered before the third day. There were features in the general appearance of the patients and in the local condition of the throat which justified a bad prognosis. Such features were the extreme pallor and prostration and the extent and character of the membrane, together with the frequent occurrence of bleeding from the throat and nose. Dilatation of the heart was always obvious before the sixth day and the tension in the radial arteries was very low. As a result of the frequent investigation of these six cases by the polygraph a great variety of heart irregularities has been collected. Premature contractions arising in the auricles or ventricles usually heralded the occurrence of some grosser type of arrhythmia. It will have been apparent that not only did each case present a particular arrhythmia, but that the rhythm of the heart frequently changed from day to day. This fact, which was emphasized in a previous publication, was corroborated in the three cases now published.

\section{Pathological.}

Autopsies were obtained in four of the six cases of this group, and the following is a brief account of the pathological findings. The hearts were removed on the day of death and were fixed in 10 per cent. formalin. After fixation the following pieces were removed for histological examination:

(1) Piece of auricular muscle-stained with (a) Sudan III and iron haematoxylin, and (b) iron haematoxylin and van Gieson's stain.

(2) Pieces of ventricular muscle-stained similarly to (1).

(3) A block of tissue from the junction of the superior vena cava and the right auricular appendix, including the sino-auricular node-stained with haematoxylin and van Gieson's stain.

(4) A block of tissue including the auriculo-ventricular node and bundle of His-stained with haematoxylin and van Gieson's stain.

1. The auricular muscle. The auricular muscle stained. with Sudan III and haematoxylin always contained some excess of fat, though never comparable to the amount found in the ventricular muscle. There was usually an increased vascularity to be detected amongst the auricular muscle fibres, and occasionally small haemorrhages were seen.

2. The ventricular muscle. In all four instances the ventricular muscle contained a very large quantity of fat. It was found diffusely throughout the muscle of both ventricles, and nearly every muscle fibre contained some fat. In some fibres it occurred as fine granules and in others as large round droplets. Some fibres obviously were more affected than others. In the cases of J. H. and E. J. there were marked patches of interstitial myocarditis, and in all cases the smallest capillaries were dilated and engorged with blood. 
3. The sino-auricular node. Throughout each node there was a considerable increase of vascularity and actual haemorrhages were fairly common. It is thought also that there was an increased infiltration with cells of the Jymphocytic class.

4. The auriculo-ventricular node and bundle of His. Though the $a-v$ node invariably showed dilated and engorged capillaries no gross histological changes could be discovered in them; and likewise there was no abnormality in the histological appearances of the auriculo-ventricular bundle or of its two main branches. Certainly there was a greater degree of vascularity in the $a-v$ bundle of J. L. than in the other three cases, and it was in this case that a period of 2:1 heart-block was detected. The relationship between the pathological picture and the types of arrhythmia will be discussed after a description of the clinical appearances of the other cases and the histological findings in the hearts of two of them.

\section{Group II.}

In this group the toxaemia was marked in all the cases and there was a copious septic membrane in the throat. Two of the cases died, on the forty-second and forty-sixth days respectively. The remainder were seriously ill for long periods, and their stay in the hospital was prolonged (114-131 days).

Robert S., aged $5 \frac{1}{2}$ years, was admitted on the fourth day of disease, and 4,000 units of antitoxin were injected on admission. The pulse-rate on admission was 124, but afterwards till the twenty-third day it did not exceed 84 . From the twenty-third day to the fortieth day, on which the child died, the pulse-rate varied between 84 and 118. Physical examination of the heart indicated that it was the seat of pathological changes, as on the 10th day there was a weakening of the first sound and displacement of the apex beat outwards.

Numerous and varying irregularities of the heart were discovered between the eighteenth and forty-sixth days, and the following table shows succinctly the varieties of arrhythmia and the days on which they were detected :--

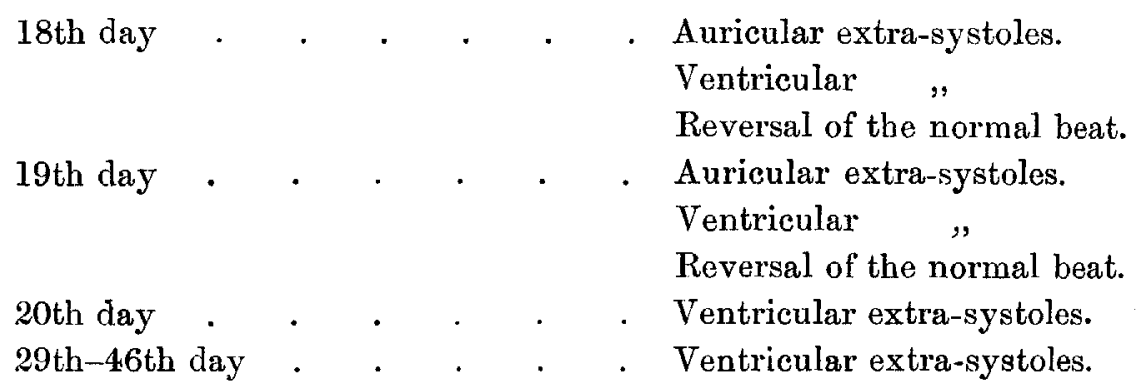

From the eighteenth day to the twentieth day auricular extra-systoles were frequent, and Fig. 5 is characteristic of their appearance. The fifth cycle is premature, and the proof that the prematurity is due to the early appearance of an auricular contraction is to be found in a study of the jugular tracing. 
Ventricular extra-systoles were very frequent from the eighteenth day onwards, and Fig. 6 is typical of the condition.

In this figure the fourth cycle from the ordinate is a premature beat, and a study of the jugular tracing shows that it is due to a premature contraction of the ventricles. The auricular rhythm is undisturbed, and the post-extrasystolic pause is fully compensatory. Occasionally ventricular and auricular extrasystoles occurred in close relationship to each other. In Fig. 7 the fourth cycle to the right of the ordinate is due to a premature contraction of the ventricle.

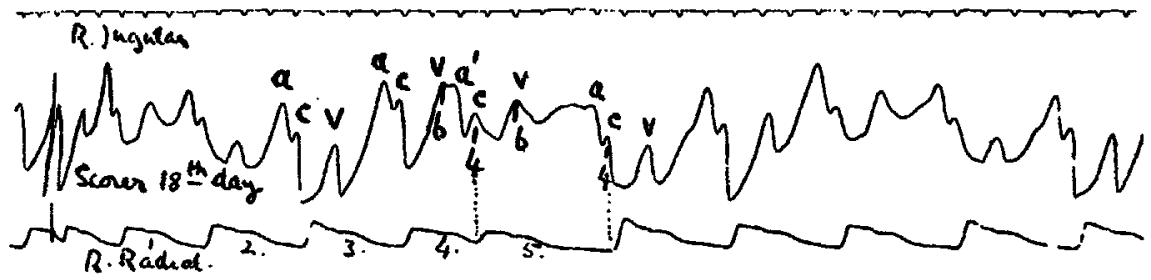

Fia. 5. The marking 6 of the fourth cycle occurs on the first apex of a broad wave. This is immediately followed by a second apex which is the $a$ wave of the premature beat.

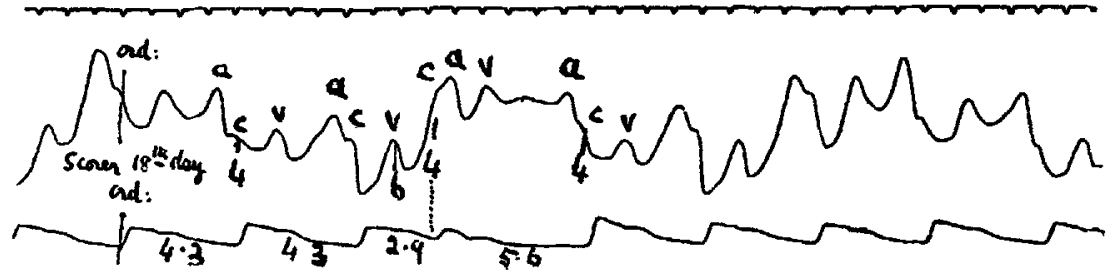

FI\&. 6. Polygraphic curves from right jugular vein and right radial axtery. The fourth cycle is premature, and measurement shows that the ventricle $(c)$ has contracted prematurely. The $c$ wave is followed by the physiological auricular contraction, and together they give rise to a large wave $c a$.

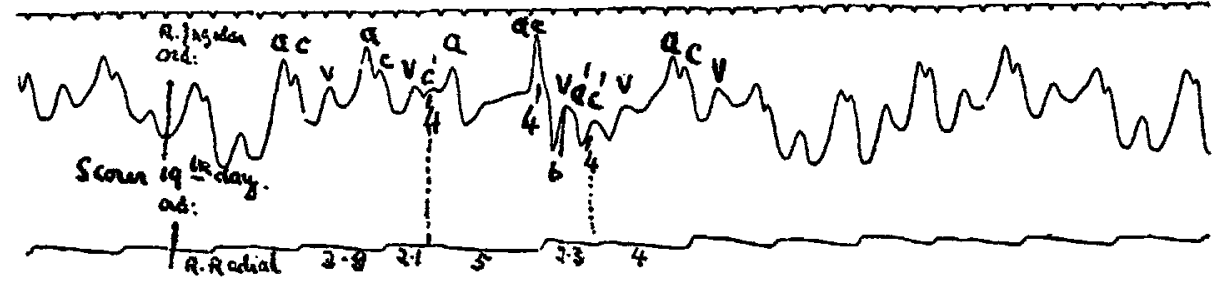

FIG. 7. The fourth cycle is premature and is due to a premature contraction of the ventricle $\left(c^{1}\right)$. In the next cycle auricles and ventricles contract together $(a c)$, followed in the sixth cycle by an auricular extra-systole.

The fifth ventricular contraction is also slightly premature and the simultaneous contraction of auricles and ventricles gives rise to a single large wave $a c$.

The sixth cycle is likewise premature and is due to a premature contraction of the auricles. The ventrieular post-extrasystolic pause is almost fully compensatory, whereas the auricular post-extrasystolic pause falls considerably short of it.

Reversal of the cardiac beat. In Fig. 8 a very exceptional mechanism is presented. The apparent $v$ wave of the fifth cardiac cycle is exceptionaliy large, 
and it is thought that a premature contraction of the auricle is included in it which meets with no response from the ventricle. The sixth cycle commences with a small wave $c$, which is followed by a large wave occurring during ventricular systole. That this is due to a contracting auricle seems almost certain from a consideration of the position of the marking 6 , which falls considerably after it. The seventh cycle is composed of a simultaneous contraction of auricles and ventricles. Thereafter the normal rhythm is re-established. It is noteworthy that the rate of the ventricles is considerably slowed during the period of most marked irregularity. This fact is accounted for by the pause which the ventricles make before they initiate their own contraction, having been disappointed of an auricular impulse from above.

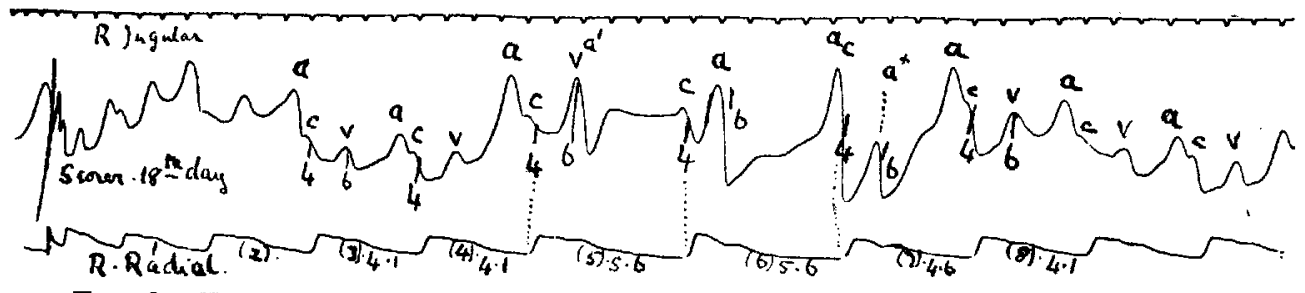

Frg. 8. The $v$ wave of the fifth cycle is so prominent that it is thought to include an $a$ wave. The sixth cycle is delayed and auricular follows ventricular contraction, while the seventh is a nodal beat.

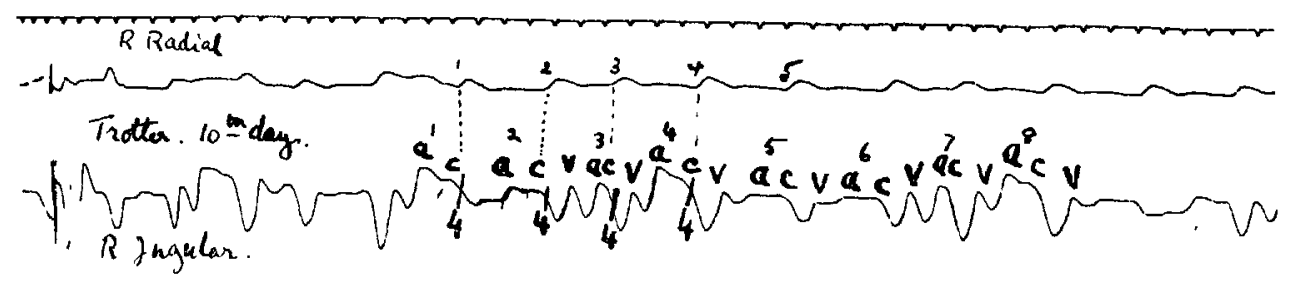

FIG. 9. Lower curve is from the right jugular vein and the upper from the right radial artery. Of the cycles which are labelled, the first two are normal acv sequences, the third contains a premature contraction of the ventricle, while in the fourth the auricular contraction

Premature beats of the ventricles persisted for some weeks, although the heart became regular before the child died on the forty-second day.

The second fatal case of this group, Margaret T., aged 5 years, was admitted on the second day of illness with an extensive membrane on both tonsils, and a purulent discharge from the nose. The pulse-rate on admission was 120 and remained rapid, varying between 90 and 144, until the fortysecond day, on which the child died. The pulse was perfectly regular until the ninth day. On the ninth and tenth days the pulse had the character depicted in Fig. 9, and the following are its characteristics :

Though the rhythm is for the most part regular, premature beats are to be seen in the radial tracing. These correspond to single waves $a^{3} c$ in the jugular curve, measurement of which shows that the ventricular wave $c$ is alone premature, while the auricular wave $a^{3}$ falls at the normal physiological moment. The premature beats are due to premature contractions of the 
ventricles, but do not interfere with the physiological rhythm. The following contraction $a^{4}$, however, is premature and in the next four cycles the same sequence of events is repeated. This type of arrhythmia was almost constantly present until the patient died on the forty-ninth day.

Both clinically and pathologically the two cases which have just been described differed considerably from all the cases included in Group I. Both R. S. and M. T. were in a clinical condition in the early stage very similar to that which will be described in the other cases of this group. Arrhythmia of the heart was the first indication that the heart was pathologically involved in both cases, and though various forms of arrhythmia appeared and disappeared, it was not surmised that a fatal termination would ensue. In both cases there was a sudden increase in the pulse-rate about the fortieth day, and in the case of M. T. pharyngeal paralysis appeared on the thirty-sixth day, and paralysis of the diaphragm on the forty-first day, and in the case of R. S. diaphragmatic paralysis was noticed on the forty-fifth day. Both children died on the day following the appearance of the diaphragmatic paralysis.

A post-mortem examination was only obtained in the case of $\mathrm{M}$. T., and the histological appearances of this heart differed very materially from the four examples which were obtained in Group I. The heart of M. T. was investigated in exactly the same manner as were the hearts in Group I. The increase of fat, which was such a noteworthy feature in the fatal cases of Group I, was completely absent in this case. Beyond some increase of vascularity throughout the whole heart, the only pathological feature that was discovered was some scattered patches of interstitial myocarditis in the ventricular muscle.

The other cases of this group were seriously ill for a considerable time, and three of them (A. S., G. R., and V. L.) had many features in common.

On admission they were all considered serious cases and showed signs of a grave toxaemia. The rate of the heart remained rapid throughout their stay in the hospital, and in one case (A. S.) the pulse-rate was 100 on discharge. In each case there were the ordinary physical signs associated with dilatation of the heart, namely, displacement of the apex beat, weakening of the first sound, and a systolic murmur at the apex.

The first case, A. S., has been reported in the paper to which reference has already been made, though the chief facts may be here repeated. A. S., aged 6 years, was adrnitted in a very toxic condition, and on the fourth day the first sound of the heart was feeble and the apex was found in the nipple line. On the twentieth day a mitral systolic murmur was heard at the apex. The rate of the heart was always rapid, and even on discharge was 100 beats per minute. The rhythm of the heart was regular until the fifteenth day, when premature beats were detected. These were observed up to the forty-first day, when the child had an attack of paroxysmal tachycardia. On this and subsequent days the patient was very collapsed and vomited frequently. She appeared almost moribund and lay in a prostrate condition until the tifty-first day, when the events of the forty-first day were repeated in almost every detail. On both these days there were short and long attacks of paroxysmal tachycardia, and one of the attacks is shown in Fig. 10.

Each cardiac cycle during the paroxysm consists of two waves in the jugular. 
tracing, $a c$ and $v$. From a comparison of this tracing with others in which electro-cardiographic records have been made it seems most probable that the exciting focus was in the auriculo-ventricular node. With this interpretation the first wave $a c$ represents the simultaneous contraction of auricles and ventricles. After the second paroxysm the child lay for three days in a state of great collapse, but gradually recovered, though the pulse-rate was still 100 when she was discharged.

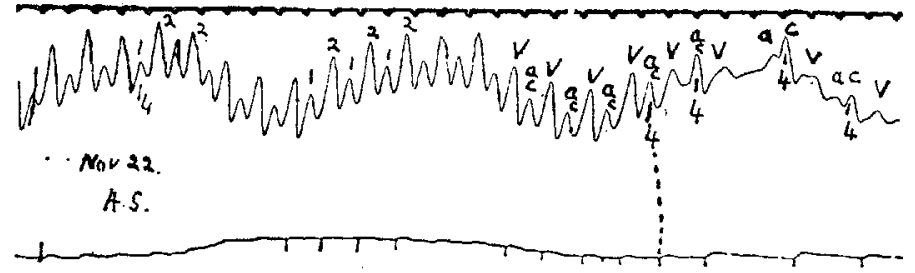

FrG. 10. Right jugular and right radial curves. The child moved her hand and this has deformed the radial curve. During the paroxysm there are two waves in each cycle $(\alpha c$ and $v)$. The paroxysm ends abruptly, and there is a post-paroxysmal pause before the normal rhythm is resumed.

Gladys R., aged 7 years, was admitted on the second day of disease, and 6,000 units of antitoxin were administered on admission and a further dose of 6,000 units was given on the third day. The throat was covered with a thick membrane and the signs of a severe toxaemia were apparent; the child was prostrate and pale. The pulse-rate on admission was 130 and remained fast until the 120th day of disease, when it fell to 96 and the patient was discharged on the 124th day with a pulse-rate of 84 .

During the first week the temperature ranged between $97^{\circ}$ and $100^{\circ}$ and was then normal for six weeks. On the fiftieth and fifty-first days the temperature rose to $99 \cdot 8^{\circ}-100 \cdot 4^{\circ}$, and the pulse-rate quickened to 144 beats per minute.

The heart was early dilated, though the rhythm remained normal until the twenty-seventh day, when ventricular extra-systoles were recorded.

This arrhythmia persisted only for a short period, and the rhythm was regular until the child was dicharged on the 124th day with a pulse-rate of 84 . Her prolonged stay in the hospital was due to the fact that she developed paralysis of the diaphragm on the forty-fifth day.

Violet $L$., aged 9 years, was admitted on the third day of illness, and both on that day and the day following 4,000 units of antitoxin were injected. The child had a toxic appearance and the tonsils were covered with membrane. The membrane separated on the sixteenth day, but fresh membrane appeared on the seventeenth day, a large piece of which came away on the same afternoon. There were evidences of cardiac dilatation from the sixteenth to the seventyfourth days, and nasal phonation was detected on the eleventh day.

The pulse-rate on admission was 128 and fell to 86 on the seventh day. The speed of the heart varied considerably throughout, and the temperament of the child may have been more responsible for this variation than any changing condition of the heart itself. The temperature on admission was $102^{\circ}$ and at the end of a week had fallen to 98.4. Except for a rise of temperature to $99^{\circ}$ on the fifty-first day the range remained constantly between $97^{\circ}$ and $98 \cdot 4^{\circ}$.

The rhythm of the heart remained perfectly regular until the sixty-first day, when ventricular extra-systoles were recorded. These were infrequent and only persisted for a few days. No other cardiac irregularity was detected. The child was discharged on the 114th day with a pulse-rate of 80 . 
The remaining cases of this group were less severe than the foregoing and in no stage of their illness did they give rise to anxiety as to the ultimate issue.

Norman C., aged 13 years, was admitted on the fourth day of illness and was injected with 6,000 units of antitoxin on the day of admission. A further dose of 4,000 units was injected the next day. On the right tonsil there was a thick, grey, white membrane which extended on to the soft palate. The submaxillary glands on the right side were considerably enlarged. The face was flushed, but there were no evidences of toxaemia such as the condition of the throat would have suggested.

The temperature on admission was $98 \cdot 4^{\circ}$. On the seventeenth and eighteenth days the temperature rose to $99 \cdot 6^{\circ}$ and $101^{\circ}$ respectively, and thereafter the range was normal. The pulse-rate on admission was 84. During the days on which there was a rise of temperature the pulse correspondingly increased in rate, but on other occasions it varied between 70 and 90 beats per minute. On the fifteenth day nasal phonation was detected. There was ciliary paralysis on the forty-eighth day. Up to the sixteenth day the heart remained regular. From the sixteenth to the thirty-first days ventricular extra-systoles were recorded, and Fig. 11 is an example.

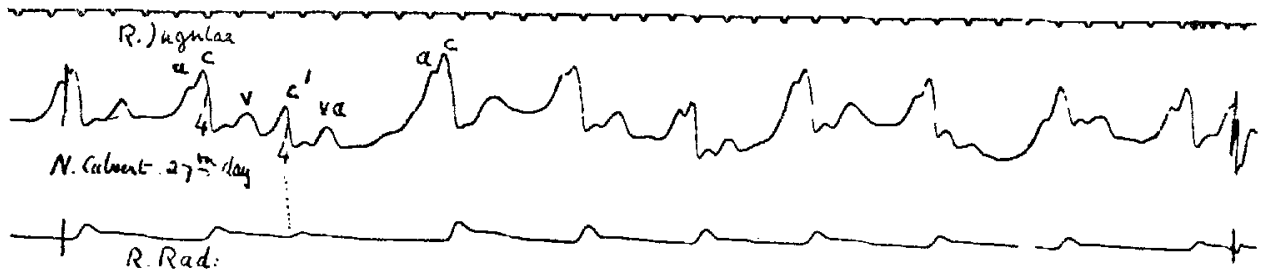

FIG. 11. Polygraphic curves from right jugular vein and right radial artery. The third cycle is premature and corresponds to $c^{1}$, a premature contraction of the ventricle. Physiological $a$ falls with the $v$ of the premature beat.

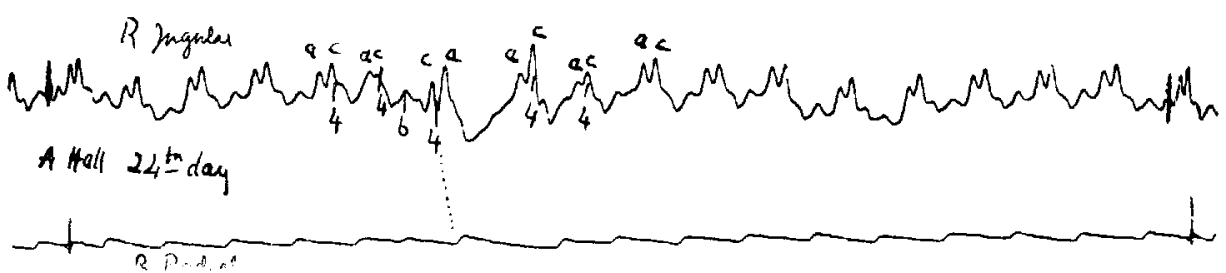

FIG. 12. Right jugular and right radial curves. The sixth eycle is premature and the prematurity involves both $a$ and $c$; this is a nodal extra-systole. The seventh cycle is a ventricular extra-systole.

After the thirty-fifth day the heart became regular and remained so until the child was discharged on the seventieth day.

Annie H., aged 9, was admitted on the sixth day of illness with membrane on the right tonsil and the right side of the uvula. 4,000 units of antitoxin were administered on the day of admission. The general condition was good and the membrane early separated. From the sixth day onwards a slight weakening of the first sound of the heart was detected. The pulse-rate on admission was 100 , and on the eighth day it had fallen to 88 . From the ninth day to the seventy-seventh day the rate never exceeded 88 beats per minute. Beyond a rise of temperature to $100 \cdot 2^{\circ}$ on the seventh day the range was normal. Nasal phonation was detected on the second day. From the twenty-fourth to the sixtieth day ventricular extra-systoles were recorded. In Fig. 12 the sixth and seventh cardiac cycles are premature. 
In the sixth cycle the auricles and ventricles have contracted prematurely and there is a considerable shortening of the $a-c$ interval. The stimulus for this almost simultaneous contraction of auricles and ventricles has probably arisen in the $\alpha-\vartheta$ junctional tissues. In the seventh cycle the ventricular precedes the auricular contraction by a considerable interval, and is followed by a compensatory pause. This is due to a premature contraction in the ventricles.

The child was discharged on the seventy-seventh day with a pulse-rate of 88 .

Charlotte M., aged 10 years, was admitted on the eighth day of illness and had received 2,000 units of antitoxin on the fifth day of illness. On admission a further dose of 4,000 units was injected. On the eighth day the child was pale and had a toxic appearance. Patches of membrane persisted on both tonsils. On the seventeenth day of illness it was noticed that the first sound of the heart had the short abrupt character of a second sound, and on the eighteenth day the pulse was found to be irregular. Polygraphic tracings taken on this day are represented by Fig. 13.

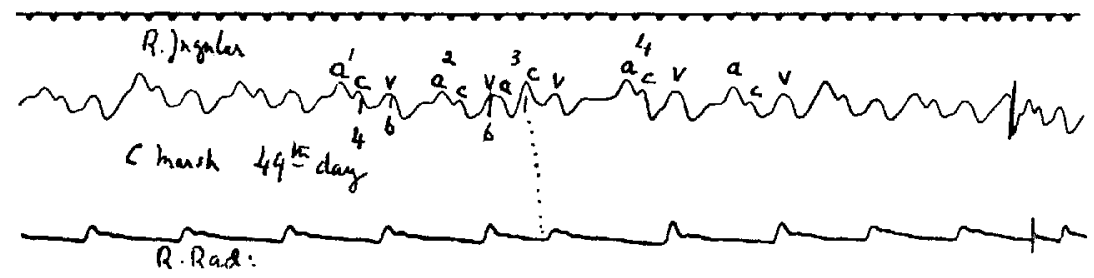

Fra. 13. Polygraphic curves from right jugular vein and right radial artery. The third cycle is premature and is due to a premature contraction of the auricles. $a^{3}$ falls on the $v$ of the previous cycle and makes that wave broad.

In this figure $a^{3}$ is premature and deforms the $v$ wave of the preceding cycle, making a much broader wave than the other $v$ waves. The compensatory pause is not complete. Nasal phonation was detected on the twenty-ninth day of illness and eiliary paralysis on the thirty-first day of illness. Throughout the ninety-two days while the child was in the hospital the pulse remained comparatively slow and the range was constantly between 68 and 99 . The temperature was normal throughout. The patient was discharged on the ninety-second day with a pulse-rate of 84 .

\section{Group III.}

There remain to be described three cases which showed many points of similarity in that the cases were not admitted to the hospital before the fifth day of illness, and had not received antitoxin before the day of admission. There was no evidence of toxaemia, and the throat condition was not severe. Certain irregularities of the heart occurred in all of them, but the heart did not reveal any abnormality by the ordinary methods of examination.

Mary $R$., aged $2 \frac{1}{2}$ years, was in the hospital for twonty-three days. On admission the pulse-rate was 80 and on the eleventh day it reached 96 . For ten days the pulse remained quick. On the ninth day it was noticed that the pulse was irregular. The irregularity was due to premature contractions of the 
ventricles, and this arrhythmia was present from the ninth to the fourteenth day. When the child was discharged from the hospital the pulse was perfectly regular at 80 to the minute.

In the case of Harry L., aged 15, premature beats of the auricles and ventricles were recorded between the twentieth and twenty-sixth days. He was admitted on the seventh day of illness, and again there was no evidence of toxaemia. The throat was congested, but there was no membrane on either tonsil. The pulse-rate was never fast and was not counted at more than 98 beats per minute. The ordinary examination of the heart did not reveal any abnormality.

In Fig. 14 it will be seen that the eighth cardiac cycle is premature, and from a consideration of the jugular curve it is evident that the prematurity is due to an auricular extra-systole.

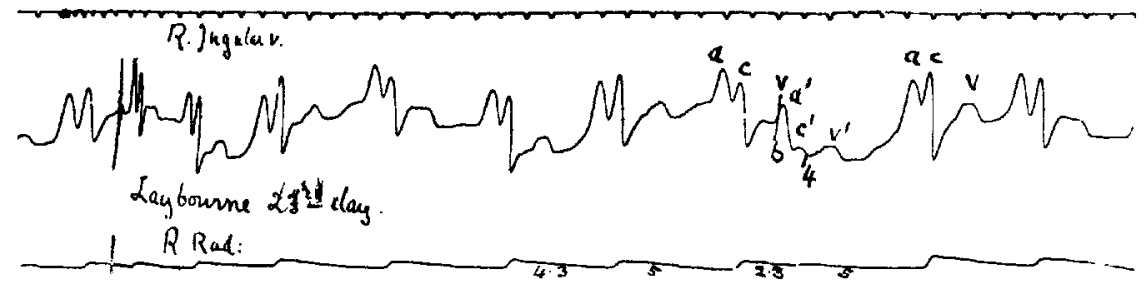

FIG. 14. Curves from right jugular vein and right radial artery. The eighth cycle is premature, and this is due to a premature contraction of the auricles.

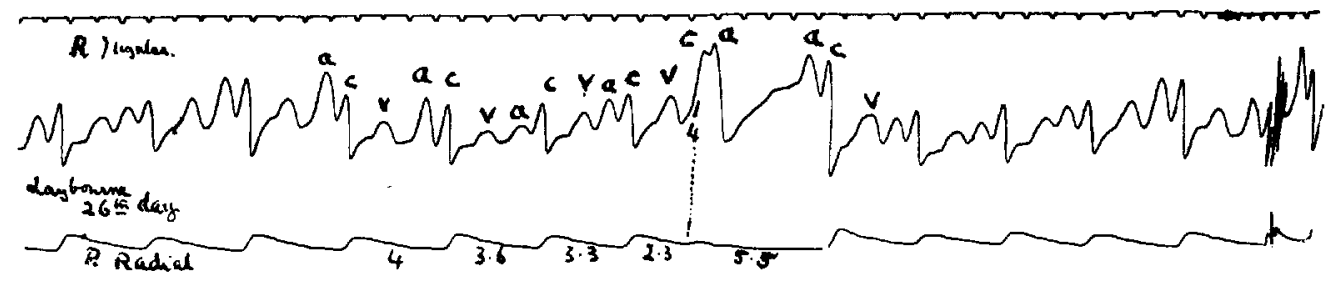

FIG. 15. Curves from right jugular vein and right radial artery. The eighth cycle is premature, and a ventricular extra-systole is evident.

The compensatory pause is not complete. Between the twentieth and twenty-sixth days ventricular extra-systoles were common, and an example is to be seen in Fig. 15.

In this figure the eighth cycle is premature, and by a consideration of the jugular curve the prematurity is found to be due to a premature contraction of the ventricle. The auricular rhythm is not disturbed and the compensatory pause is more than complete, the cycle previous to the premature beat and the premature beat itself measuring $7 \cdot 8$ fifths of seconds, while no other two normal cycles measure more than $7 \cdot 6$ fifths of seconds. During the last fortnight there was no irregularity of the pulse at all, and the boy was discharged cured.

The last case of the series, George C., aged 17, was admitted with small patches of membrane on the left tonsil and his general condition was good. $\mathrm{He}$ was in the hospital for twenty-four days, and on the fifteenth day ventricular 
extra-systoles were recorded (Fig. 16). The pulse is slow, and at the sixth cycle a premature $c^{1}$ has deformed the $v$ wave of the fifth cycle. The sixth auricular contraction, $a^{6}$, is plainly seen as an isolated wave, and there has been no ventricular response. The seventh auricular contraction is premature.

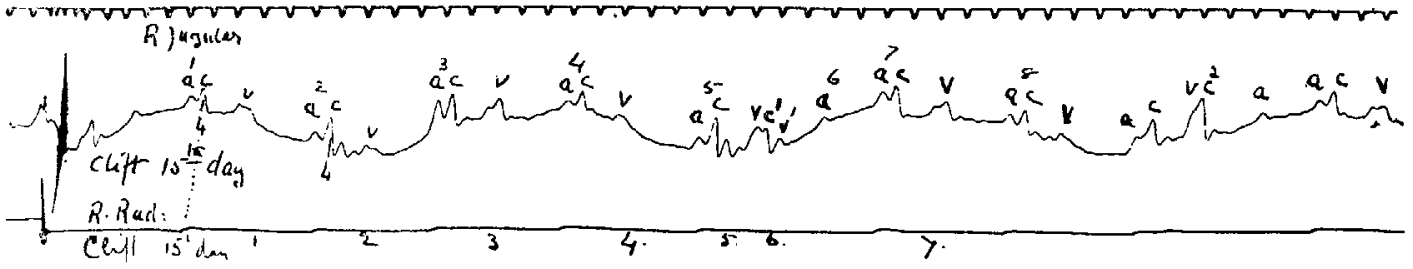

Fig. 16. Jugular and radial curves. The sixth cycle, $c^{\mathbf{l}} v^{\mathbf{1}}$, is premature and is due to a ventricular extra-systole. The physiological $\left(\alpha^{6}\right)$ is seen as an isolated wave.

\section{Summary.}

At the commencement of this investigation it was not anticipated that a systematic polygraphic study in cases of diphtheria would reveal such a variety of irregularities of the heart, and the accompanying table, Table II, shows the types of arrhythmia which have been recorded in the cases which are now described (15) and in those whose detailed description appeared elsewhere (3).

Although the pathological changes in four cases of Group I were very marked, it is impossible to correlate the pathological lesions with the individual instances of arrhythmia of the heart. Irritative and destructive processes are taking place simultaneously and result in excitation or depression of the various functions of the heart muscle. For instance, it is impossible to state whether a nodal rhythm depends on hyper-irritability of the $a-v$ node, or a depression of the $s-a$ node allowing the lower node to usurp the function of pacemaker of the heart, which belongs properly to the upper node. Further, we have shown that the types of arrhythmia in each individual case may vary from day to day, a variation which is in all probability dependent upon varying and progressive pathological changes in the heart muscle and its nerves. It may be asserted, however, that any form of arrhythmia of the heart (except sinus arrhythmia) in diphtheria indicates that the heart muscle or nerves are involved in a pathological process, however mild the illness may otherwise appear to be, and that special precautions are necessary to keep the patient recumbent.

(The pathological preparations were made in the Pathological Department of the Durham University College of Medicine.) 


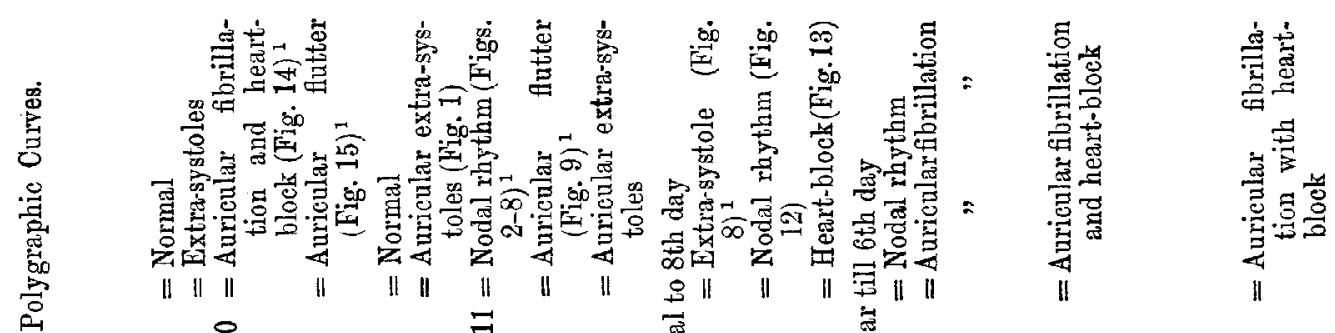

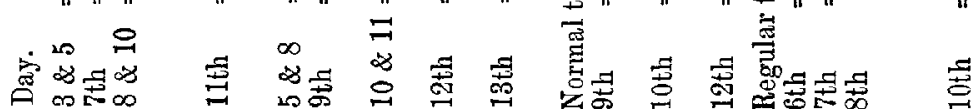

委
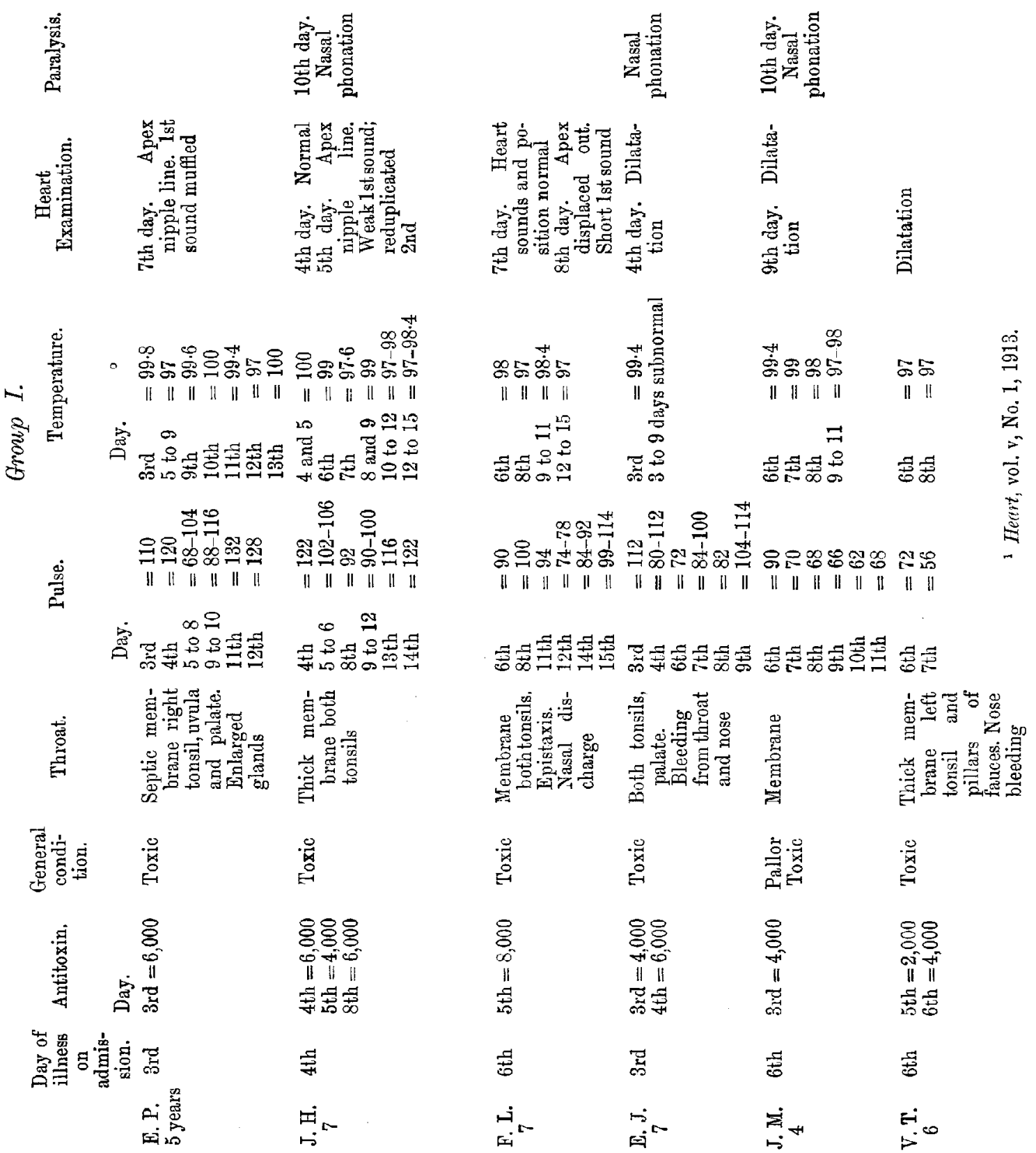


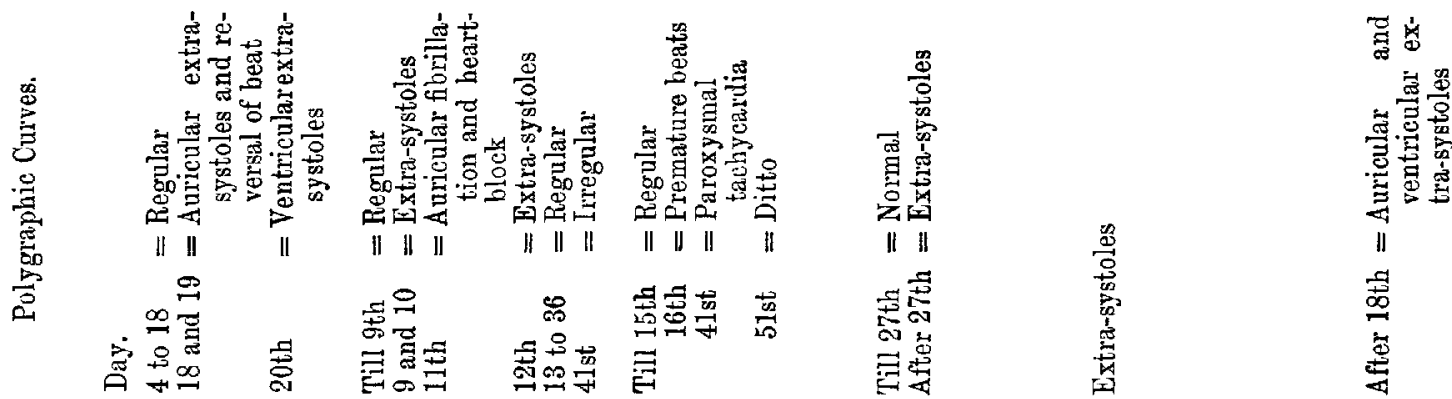

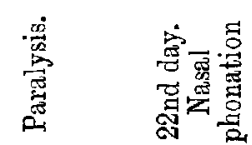
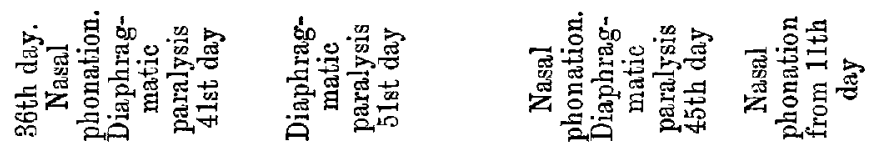

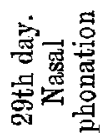

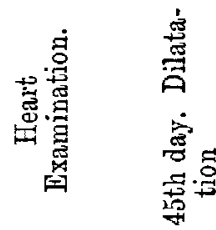

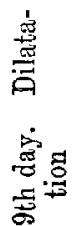
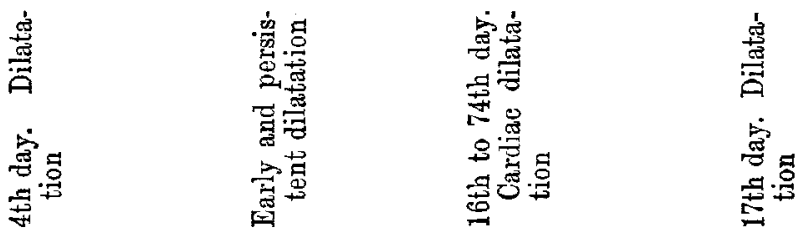

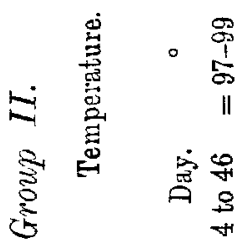
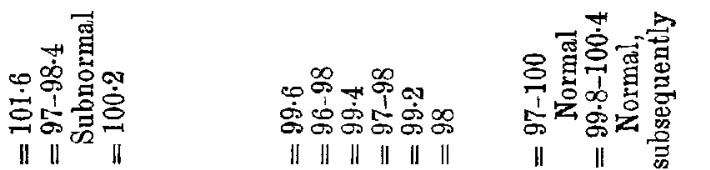

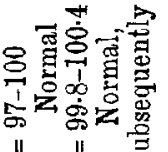

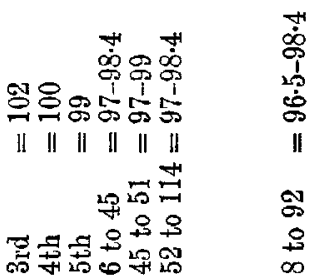

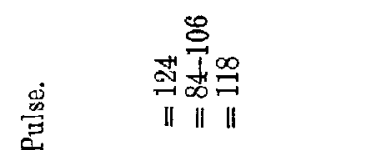

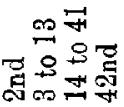

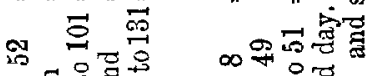

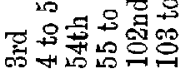

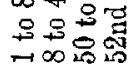

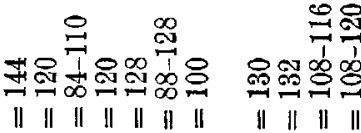

|| || || || || || || || || || ||

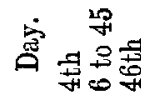

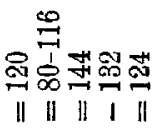

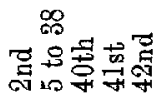

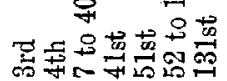

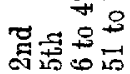

产方象官

|| || || || ||

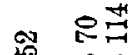

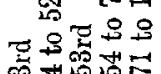

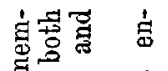

营

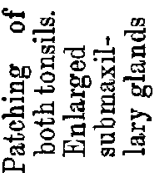

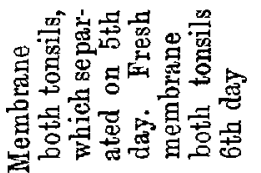

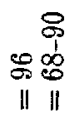

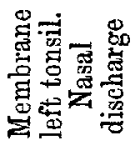

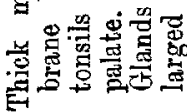

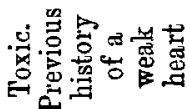

붕

영

竜 总

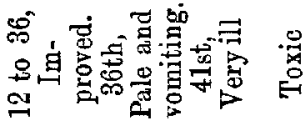

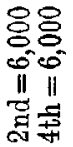

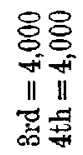

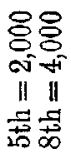

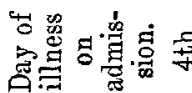

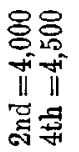

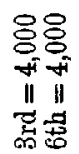

్ㅗㅇ

ํㅗํ

్ㅗํ

क्ष̉

串

Ei

कें

مَ

म்

놀옹 

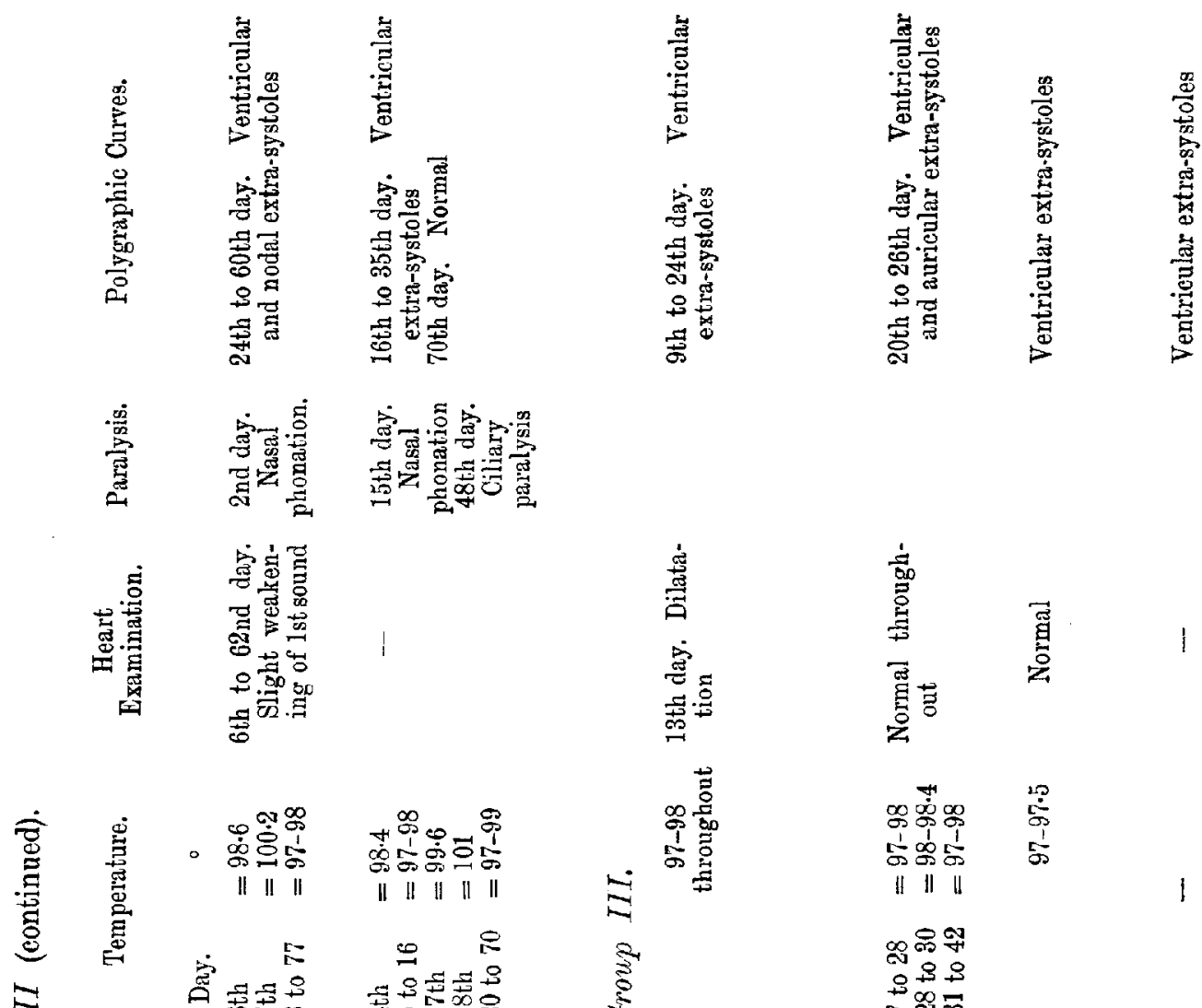

$$
\text { - }
$$

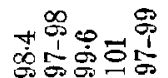

|| || || || ||

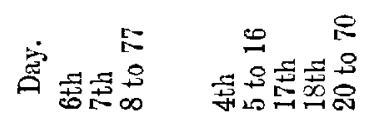

हैํำ

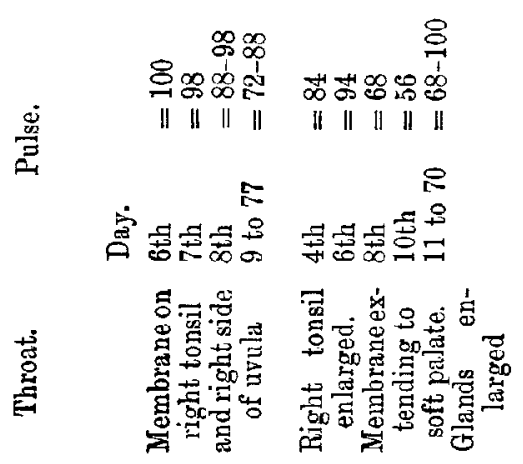

密: ‘்

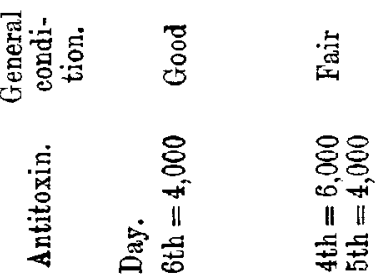

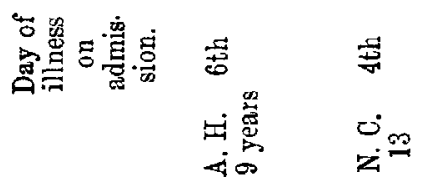

$$
\text { 出蒂 }
$$

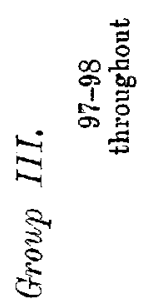

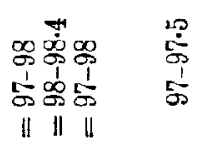

㖂架

옹요

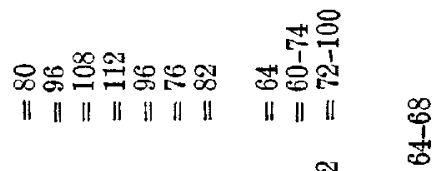

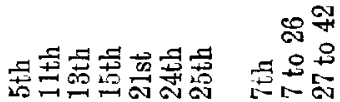

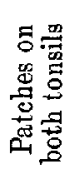

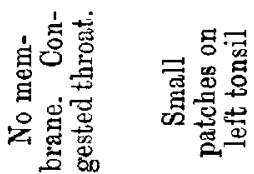

تั:

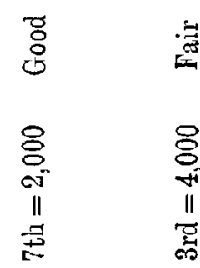

폼

पंत

$\vec{z}$

舟舟

$\dot{0}=\dot{0}$ 
TABLE II

Auricular Extra-systoles (5).

Case. Group. Onset. Duration.

$\begin{array}{lrll}\text { J. H. } & \text { I } & \text { 9th day } & 4 \text { days } \\ \text { F. L. } & \text { I } & \text { 9th } " & \text { 1 day } \\ \text { R. S. } & \text { II } & \text { 18th } ", & \text { days } \\ \text { C. M. } & \text { II } & \text { 18th } " & 74 \text { days } \\ \text { H. L. } & \text { III } & \text { 20th " } & 6 \text { " } \\ & & \text { Ventricular Extra-systoles (11). }\end{array}$

E. Y.

G. C.

H. L.

M. R.

N. C.

A. $\mathrm{H}$.

R. S.

G. R.

C. $\mathrm{M}$.

M. T.

V. L.

III
III
III
III
II
II
II
II
II
II
II

$?$

20 th day

9 th and 24th days

16 th day

24th day

18 th and 19th days

27 th day

18th day

9 th and 12 th days

6 days

2 ,"

18 ,"

$36 "$

26 ",

short period

1 day

2 days

short period

Auricular Flutter (2).

F. P.

J. H.

I 11th day

12 th ,"

1 day

1 ,

Paroxysmal Tachycardia (1).

A. S. II 41 st and 51 st days $\quad 2$ days $^{2}$

Auricular Fibrillation (1).

E. J.

I

7th and 8th days

2 days

Auricular Fibrillation and Heart-block (3).

V. T.

E. P.

J. M.

I

I

7th day

8 th and 10th days

7 th day

1 day

2 days

Heart-block (1).

F. L.

I

12 th day

1 day

Nodal Extra-systoles (1).

A. H.

II

24th day

36 days

Nodal Rhythm (3).

E. J.

J. M.

E. P.

R. S. 6th day

10 th day

8th and 10th days

Reversal of Nodal Beats (1).

II 18th day
1 day

1 ,"

2 days

1 day

\section{DESCRIPTION OF PLATES.}

Piate 1. Ventricular muscle stained with Sudan III and haematoxylin. Accumulation of fat in muscle fibres seen in four cases of Group I.

Platre 2, Fig. 1. Microphotograph of ventricular muscle stained with haematoxylin and Sudan III. The dark areas are muscle cells more or less completely filled with fat.

FIG. 2. Ventricular muscle showing a central patch of myocarditis. 


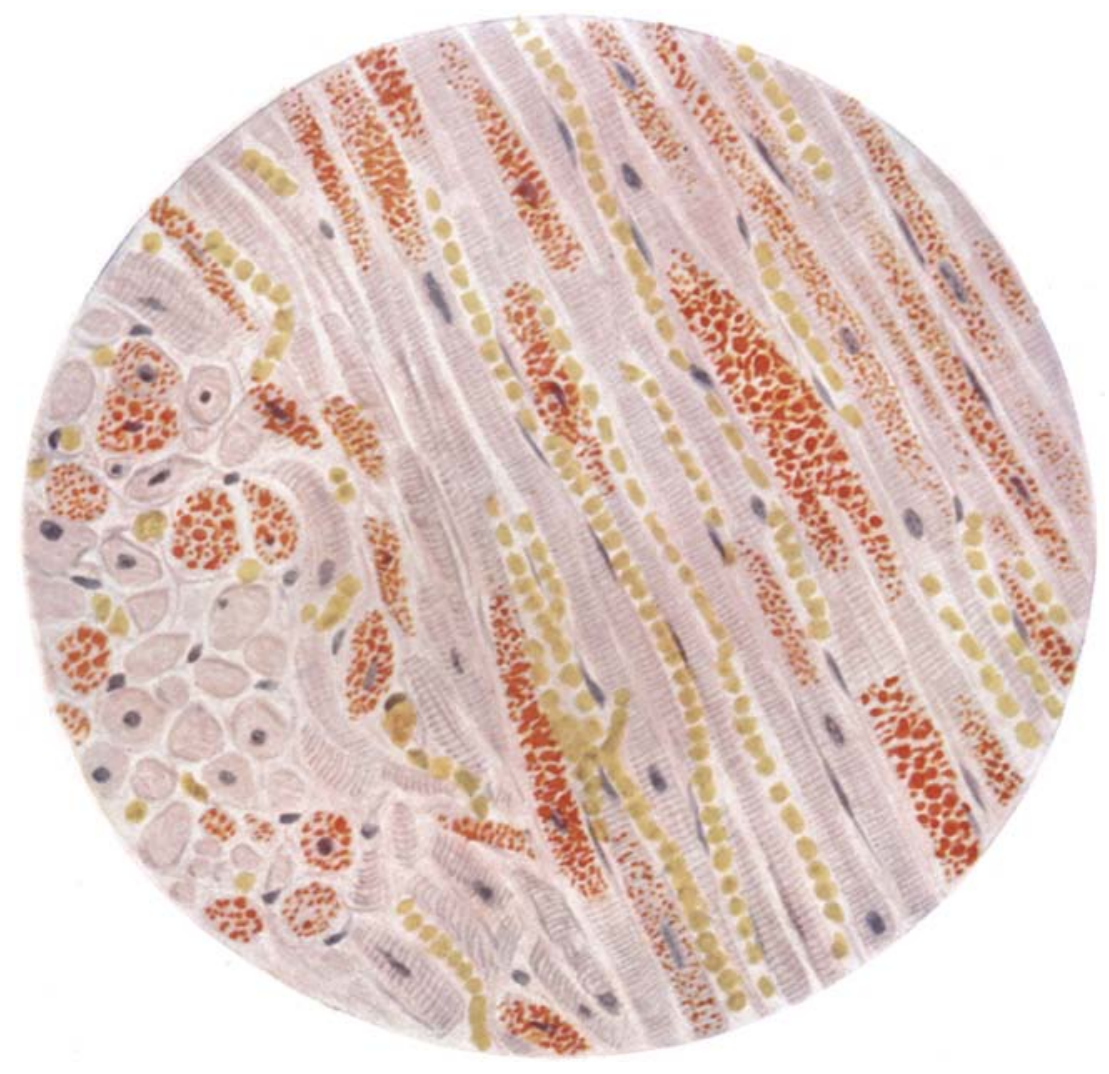


Vol. $8 \quad P l .2$

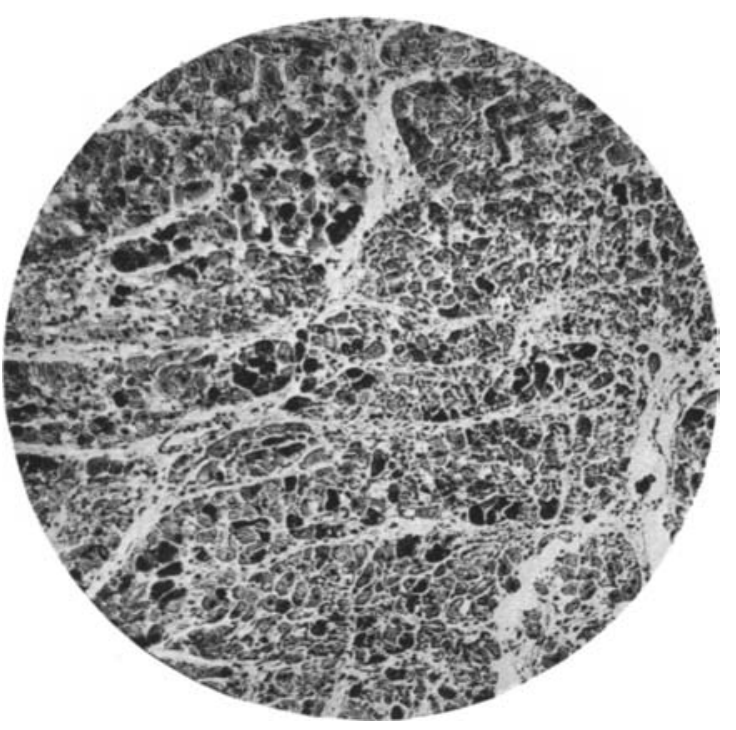

FIG. 1

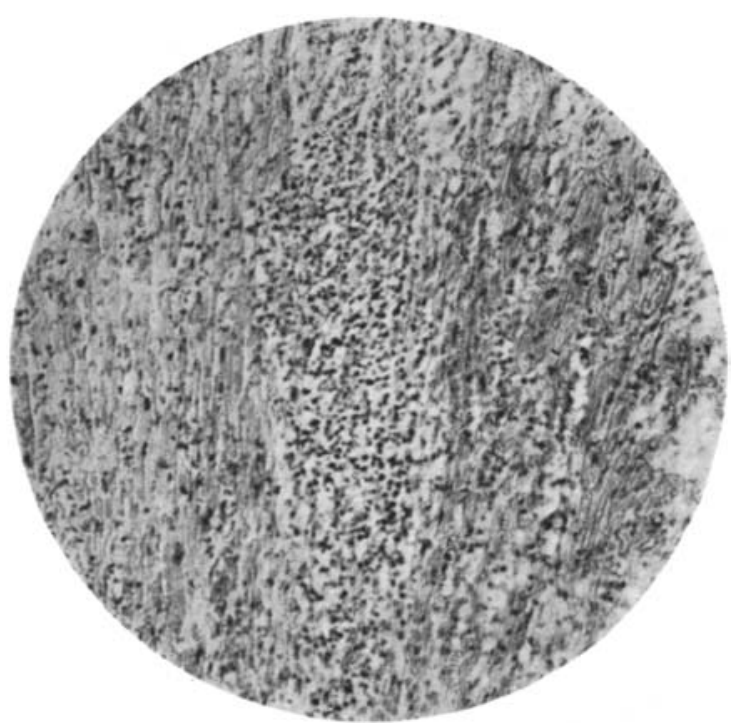

Fig. 2 